1 Dogs (Canis familiaris) adjust their social behaviour to the differential role of inanimate

3

\title{
interactive agents
}

Eszter Petró ${ }^{1}$, Judit Abdai ${ }^{1}$, Anna Gergely ${ }^{1,2}$, József Topál ${ }^{2}$ and Ádám Miklósi ${ }^{1,3}$

(n)

\section{Affiliations}

${ }^{1}$ Department of Ethology, Eötvös Loránd University, Budapest, Hungary

${ }^{2}$ Institute of Cognitive Neuroscience and Psychology, Hungarian Academy of Sciences, Budapest, Hungary

${ }^{3}$ MTA-ELTE Comparative Ethology Research Group, Budapest, Hungary

*Corresponding author: petroeszti1989@gmail.com

Keywords: dog, problem-solving, social interaction, inanimate agent

\begin{abstract}
Dogs are able to flexibly adjust their social behaviour to situation-specific characteristics of their human partner's behaviour in problem situations. However, dogs do not necessarily detect the specific role played by the human in a particular situation: they can form expectations about their partners' behaviour based on previous experiences with them. Utilizing inanimate objects (UMO - Unidentified Moving Object) as interacting agents offer new possibilities for investigating social behaviour, because in this way we can remove or control the influence of previous experience with the partner. The aim of the present study was to investigate whether dogs are able to recognize the different roles of two UMOs and are able to adjust their communicative behaviour toward them. In the learning phase of the experiment dogs were presented with a two-way food-retrieval problem in which two UMOs, which differed in their physical appearance and abilities, helped the dog obtain a piece of food in their own particular manner. After a short experience with both UMOs, dogs in the test phase faced with one of the problems in the presence of both inanimate partners. Overall, dogs displayed similar levels of gazing behaviour toward the UMOs but in the first test they looked, approached and touched the relevant partner first. This rapid adjustment of social
\end{abstract}


behaviour toward UMOs suggests that dogs may generalize their experiences with humans to other unfamiliar agents, and are able to select the appropriate partner when facing a problem situation.

\section{Introduction}

An intriguing problem in animal communication is whether and how individuals communicate their needs or goals to their companions. In the case of cooperative activities an individual may be facing an unsolvable problem, and it is necessary to solicit the partner's assistance in order to achieve its goal. For example, Melis et al. (2006) reported that chimpanzees are skilful in recognizing the situations in which collaboration is necessary and in determining who is the best collaborative partner.

Efficient solicitation of potential collaborators can be beneficial to both partners, and may also strengthen the inter-individual relationship. In some species such soliciting behaviour consists of a directional component which is related to the external target/problem and an attentional-getting component that directs the attention of the partner to the solicitor (e.g. Miklósi et al. 2000). For example, dogs indicate the location of a hidden target (e.g. food) to humans by gaze alternations between the hidden target and the human in a way that is functionally similar to infant behaviour in comparable situations (Miklósi et al. 2000; Gaunet 2008, 2010).

Virányi et al. (2006) run a nonverbal problem solving test in which dogs and 2.5 years old infants solicited help from a human helper by indicating the location of an out-of-reach desired toy and the tool needed to obtain it. In the four experimental conditions the helper was either present or absent during hiding of the toy and the tool and thus she knew only the location of the toy, the location of the tool, both or neither of them. Both dogs and children signalled the place of the toy more frequently when the helper was absent during the hiding compared to the condition when the helper was present. Kaminski et al. (2011) noted that dogs become more excited when the helper left them alone in the room and this could have led to a higher level of soliciting behaviours toward the returning helper. Although this cannot explain why dogs exhibited more frequent signalling only to the object that the helper had not witnessed being hidden, more recently it has been argued that dogs' differential communicative behaviour toward the helpers might be the result of experience with them during the training phase of the experiment (Gaunet and Massioui 2014). 
Gaunet and Massioui (2014) tested dogs and 1-year-old infants in a similar problem solving test to see whether they increase communicative signalling toward a human helper (owner or caregiver) if she was absent during the hiding of the target. The experimenter placed an out of reach toy either above or under one of two containers in the presence of the dog/infant and either in the presence or absence of the helper. Both dogs and infants tended to solicit help in both conditions and no differential communicative behaviour was reported in any of the test situations. Importantly however subjects were called upon by the helper to locate the toy, thus both dogs and infants may have simply responded to the imperative order.

The above mentioned studies indicate that dogs behave in ways which are at least functionally similar to that of 1-to 2-year-old infants, and these communicative interactions between humans and dogs show a close behavioural correspondence to mother-infant interactions. Note however, that there are doubts as to whether the cognitive mechanisms underlying the behavioural similarities in these species are the same (e.g. Lakatos et al. 2009).

A recent study (Horn et al. 2012) suggested that dogs may be able to flexibly adjust their social behaviour to situation-specific characteristics of their human partner's behaviour in a problem solving situation. Dogs could learn that each of the two human partners (filler and helper) can solve one of two different problems. In the training phase dogs learned to use efficiently a rotatable disc food-container to obtain 6 pieces of food. This apparatus was equipped with a blocking mechanism that when activated, blocked the rotation of the disc, thus only 3 pieces of food were accessible to the dogs. The filler re-baited the apparatus with food if the dogs emptied it by eating all food pieces and the helper unblocked the apparatus if it got blocked during the dogs' manipulation. In the test phase dogs approached the helper first independently whether the apparatus was blocked or empty, but spent more time near the filler when the apparatus was empty. The authors argued that dogs recognized the specific role of the filler but not the helper. However, it is unclear whether the dogs' behaviour indicated a communicative intent (for the behavioural criteria see Gaunet and Deputte 2011) or the dogs had an expectation toward the filler to bring food without recognising the filler's role in refilling the apparatus.

Other observations also show that dogs may have limited capability to solve physical problems, for example, dogs failed to recognize the function of intermediate steps in a more complex sequences of action that are only indirectly linked to getting the reward (Virányi et al. 2006).

The Horn et al. (2012) study has further limitations. (1) Dogs have expectations about the humans' actions due to their previous experiences with them. Thus they more or less prepared 
to recognize the role of the filler, because getting the food from humans is a daily event. In contrast, the unblocking by the helper was an unusual action for the dog. (2) Dogs may have had difficulties recognising the nature of the physical problem they were exposed to (i.e. the blocking mechanism) therefore they were not able to distinguish between the partners based on their specific roles. (3) Dogs had unbalanced exposure to the partners, because only the helper was interactive with the dog (the helper encouraged the dog to manipulate the apparatus) in the training phase, but the dogs had more trials with the filler (who always refilled the apparatus) in the test phase.

Whether or not dogs are able to choose their potential collaborators based on the partner's problem solving competence and/or its willingness to cooperate still waits further clarification. It is increasingly assumed that the use of interactive robots offers new possibilities for studying inter-specific social behaviours (e.g. Kubinyi et al. 2004; Krause et al. 2011, Ladu et al. 2015, Spinello et al. 2013) because the uncontrolled effects of previous experiences can be eliminated, a robot's abilities and behaviour can be manipulated independently of its embodiment and the experimenter can have more control over the robot's behaviour compared to a living partner. In an earlier research (Gergely et al. 2013), we found that in a problem situation dogs show similar behaviours toward an inanimate moving object (UMO - Unidentified Moving Object) as they display toward a human whose behaviour matched that of the UMO. However, the interactive behaviour of the dog emerged faster and became more elaborated when the UMO was endowed with features typically linked to animacy (eyespots, self-propelled motion and contingent reactivity).

The aim of the present study is to investigate dogs' ability to show differential soliciting behaviour toward two physically dissimilar UMOs which assisted them in getting food by solving different problems. We used a modified version of the experimental protocol published by Horn et al. (2012) replacing the human helpers with UMOs. We aimed to find out whether dogs interact with the respective agent which was observed to be able to solve the problem. We predicted that in the test trials dogs should gaze, alternate their gaze, approach and touch the UMO which assisted them in respective context during previous encounters.

Such discrimination would rapidly emerge in dogs, because they have also been shown to learn about rules rapidly in cooperative social contexts even when interacting with unfamiliar human partner (Topál et al. 2005) and also with conspecifics (Brauer et al. 2012).

\section{Materials and Methods}


Subjects

Fifty-eight adult pet dogs were recruited from the Family Dog database of the Department of Ethology, Eötvös Loránd University. We excluded $10 \mathrm{dogs}$ because during the familiarisation phase or the first 3 trials of the learning phase they wanted to leave to room, did not took the reward from the UMOs or showed avoidance toward one of the UMOs. The remaining 48 dogs (mean age \pm SD: $3.7 \pm 2$ years, 33 females, 25 males from different breeds) were randomly assigned to one of four experimental conditions (see Table 1). Subjects were allowed to participate only if they could be motivated with food. All subjects participated only in one of the four conditions:

Our experiment is based on non-invasive procedures for assessing dogs' behaviour. Noninvasive studies on dogs are allowed to be done without any special permission in Hungary by the University Institutional Animal Care and Use Committee (UIACUC, Eötvös Loránd University, Hungary). The currently operating Hungarian law “1998. évi XXVIII. Törvény” (The Animal Protection Act) defines experiments on animals in the 9th point of its 3rd paragraph (3. 1/9.). According to this definition our non-invasive observational study does not fall in the category of animal experiments. Our experimental procedure was consistent with the ASAB/ABS guidelines on the use of animals as described in "Guidelines for the treatment of animals in behavioural research and teaching".

The owners responding to our advertisement at the department's home page (http://kutyaetologia.elte.hu) volunteered to participate and provided written consent.

\section{Apparatus}

Dogs were tested at the Department of Ethology, Eötvös Loránd University in a $4.5 \mathrm{~m} \times 3.5 \mathrm{~m}$ testing room. In this experiment we used a remote-controlled (RC) car (\#32710 RTR SWITCH, $28 \mathrm{~cm}$ x $16 \mathrm{~cm}$ x $13 \mathrm{~cm}$ ) and a remote-controlled crane (Hobby Engine Premium Label RC Crane Truck $2.4 \mathrm{GHz}, 65 \mathrm{~cm} \times 17 \mathrm{~cm}$ x15cm) as UMOs (see Figure 1a). The car was controlled by Experimenter 2 who was standing in the corner of the lab and the crane was controlled by Experimenter 3 who was standing in the other corner of the lab. The UMOs were parking outside of the room and they could enter the room through two guillotine doors. Experimenter 2 opened the guillotine door for Experimenter 3 while she was driving the UMO and Experimenter 3 opened the door for Experimenter 2 (Figure 2). Throughout the experiment Experimenter 2 and Experimenter 3 did not interact with the dog. 
An opaque wooden box ( $80 \mathrm{~cm}$ x $48 \mathrm{~cm} \times 38 \mathrm{~cm})$ was used as a hiding location. There were two holes (20 X $20 \mathrm{~cm}$ (front) and $12 \times 12 \mathrm{~cm}$ (top) openings with closable lids) on the box; one on the top and one on the front side. The food was placed in plastic bowl $(7 \mathrm{~cm} \mathrm{x} 7 \mathrm{~cm})$ which could be taken out by the UMOs from the box by the means of magnets. The UMOs differed in their physical abilities: the car obtained the food through the front hole and the crane got the food from above through the hole on the top the crane had a magnet mounted on its arm that could connect to a screw that was attached to the bowl, while the car had magnets on its front and the bowl belonging to it had magnets on its side (see Figure 1.).

\section{Procedure}

Familiarisation phase: (1) The owner and the dog entered the room, the owner released the dog and the dog could explore the room. The wooden box had been placed already in the centre of the room but the UMOs were not present. Experimenter 2 and 3 had already been stood in the room in their predetermined location (the opposite side as the UMO that was controlled by them, i.e. same side as the guillotine door they operated). Next the owner sat down at a predetermined location $(\mathrm{O})$ and held the dog in front of him/her at a distance of $2 \mathrm{~m}$ from the box (Fig. 3).

(2) Experimenter 1 entered the room and put a piece of food into a bowl in front of the dog which the dog could eat. Then Experimenter 1 put a piece of food into the bowl and placed the bowl either next to the front hole or next to the top hole depending on which UMO entered the room first (car - front hole, crane - top hole). The proper UMO entered the room and took the bowl to the dog that was allowed to eat the food. Then the UMO went out from the room at the same door.

(3) Experimenter 1 placed the baited bowl at the other location (next to the front or top hole respectively) and the other UMO entered the room and took the bowl to the dog (see also Figure 2). The order of the UMOs was counterbalanced between subjects.

\section{Learning phase:}

In the problem situation two UMOs (car and crane) helped the dog to obtain a piece of food which was placed at an inaccessible location inside the box.

(1) Experimenter1 entered the room with the bowl and one piece of sausage in her hands. She showed the food to the dog then took it into the bowl.

(2) Experimenter 1 placed the bowl through one of the two holes into the box, closed the lid on the other hole on the box and left the room. The owner took off the leash and encouraged 
the dog to get the inaccessible food from the box. After 30 seconds the owner called the dog back.

(3) The UMO which was capable for taking out the bowl through the currently open hole on the box entered the room. The guillotine door was opened for this UMO by the Experimenter ( 2 or 3 ) who controlled the other UMO by the means of hidden strings. The UMO took out the bowl from the box and carried it to the dog who was allowed to eat the food (see Figure 2). Both UMOs helped the dogs to get the food for 5-5 times. Two different orders for these interactions were used (car=1, crane =2): 1-2-1-2-2-1-2-1-1-2 or 2-1-2-1-1-2-1-2-2-1.

Test phase: After the learning phase the owner and the dog left the room for 2-3 minutes with Experimenter 1, while Experimenter 2 and 3 placed the UMOs to the front of one of the guillotine doors (see Figures 2 and 3). Then the owner and the dog (on leash) entered the room, the owner sat down at his/her predetermined location and held the dog in front of $\operatorname{him} /$ her.

Then Experimenter 1 entered the room with the bowl and one piece of sausage in her hands. She showed the food to the dog, put it into the bowl and hid the bowl into the box through one of the holes (front hole or top hole). She closed the lid of the other hole and left the room. The owner and the dog (on leash) went to the box and the dog was allowed to sniff into the box through the open hole. Next they went back to their predetermined location and the owner sat down. Then the owner took the dog off leash and encouraged it to move freely in the test room for 30 seconds.

We observed the dogs' behaviour when they faced one of the two problems in the presence of both passive UMOs. Subjects participated in two test trials in which either the top hole or the front hole was baited. The UMOs were placed next to the same or the opposite door which they used to enter the room during the Learning Phase. Thus the order of trials across the subjects was counterbalanced for the hole that was opened and the location of the UMO as well (see Table 1).

After the 30 second the appropriate UMO started to move and took the bowl with the food to the dog.

\section{Behavioural variables and data analysis}

All trials were videotaped and dogs' behaviour during the $30 \mathrm{~s}$ of free movement was analysed later with Solomon Coder 12.06.06 (András Péter http://solomoncoder.com). 
233 Below is the list of behavioural units coded during the test trials. Except 'Looking at the

234 UMO' and 'Gaze duration' all other variables were measuring occurrence/non-occurrence.

235

236

237

238

239

240

241

242

243

244

245 The Cronbach alpha was 0.934 .

First look (0/1): The dog looks first at one of the UMOs (car or crane) after the owner released the dog.

First approach (0/1): The dog approaches one of the UMOs within $1 \mathrm{~m}$ with his nose.

First touch (0/1): The dog touches one of the UMOs with its muzzle and paw.

Score 1 was given if the dog interacted (looked, approached, touched at) the appropriate partner (i.e. the car when the front hole was open; the crane when the top hole was open), and score 0 was given if the dog interacted with the inappropriate partner (i.e. the car when the front hole was open; the crane when the top hole was open).

Looking at the UMO (duration, s): looking duration at one of the UMOs.

Gaze alternation: number of gaze shifts between one of the UMOs and the box (place of food) directly (The criteria for gaze alternation was one second delay between the two gazes and looking at the UMO or the box was maximum 2 second long).

For statistical analysis we used IBM SPSS 21. First, we examined whether dogs chose the appropriate partner in the test phases (first look, first touch, first approach) using one-sample Binomial test ( 0.5 chance level) and one-sample Wilcoxon test was used to analyse whether dogs looked more at the appropriate partner in the test phase (proportion of the looking duration at the appropriate UMO: looking duration at the appropriate partner/sum of the looking duration).

Next we analysed the number of gaze alternations with GLMM for Binomial distribution in order to examine the effect of condition (same door or changed door), test partner (car vs. crane) and the repetition of test trials. We calculated the ratio of looking at the car and crane from the Looking at the UMO (duration) variable and we analysed it with GLMM for Normal distribution.

\section{Results}

First, we examined whether dogs choose the appropriate UMO in the test phase when they faced one of the problems in the presence of both passive UMOs. One-sample Binomial test 
showed that dogs looked first $(P=0.0001)$, approached first $(P=0.009)$ and touched first $(P=0.003)$ the appropriate UMO according to the problem situation in the first test trial but not in the second trial (first touch $P=0.770$; first approach $P=0.626$; first touch $P=1.00$ ). Next we tested whether other factors may have influenced the dogs' choice. Binomial GLMM showed no effect in the case of the First approach $\left(N=78\right.$; Condition: $\mathrm{F}_{1,74}=3.859 P=0.053$; Partner: $\mathrm{F}_{1,74}=0.036 P=0.849$; Trial: $\mathrm{F}_{1,74}=3.566 \mathrm{P}=0.063$ ) and First touch (N=67; Condition: $\mathrm{F}_{1,63}=1.083 \mathrm{P}=0.302$; Partner: $\mathrm{F}_{1,63}=0.366 \mathrm{P}=0.548$; Trial: $\left.\mathrm{F}_{1,63}=3.582 P=0.054\right)$. However in the case of the First look at the appropriate partner Condition (same or changed door) (Condition: $\mathrm{F}_{1,94}=4.371 P=0.039$ ) and repeated testing trials (Trial: $\mathrm{F}_{1,94}=6.695 \quad P=0.011$ ) reduced the looking at the appropriate partner, but there is not effect of the partner $(N=94$ Partner $\mathrm{F}_{1,94}=0.161 ; P=0.689$ ). Note that the number of individuals is different for each measure because some did not touch or approached either of the UMOs during the test.

We also examined whether dogs looked longer at the appropriate UMO in the test phases. One-sample Wilcoxon signed rank test showed that dogs looked longer at the appropriate UMO $(N=48 ; \mathrm{T}(+)=872 P=0.004)$ in the first trial, but not in the second trial $(N=48 \mathrm{~T}(+)=411$ $P=0.69)$.

The analysis of Looking time proportion toward the partner with GLMM (Normal distribution) and the Number of gaze alternations (Binomial distribution) did not show significant effects indicating also that in general dogs had no preference to look at the car or the crane (Looking time proportion: $\mathrm{F}_{5,90}=0.39, P=0.85$; Number of gaze alternations: $\mathrm{F}_{5,70}=$ $0.604, P=0.697)$.

\section{Discussion}

We found that dogs chose the appropriate UMO for obtaining the food, as they approached, touched and looked first at the interacting agent which was able to retrieve the reward in the respective context. However, dogs looked longer at the appropriate partner only in the first test trial. This effect emerged because in the test the UMO did not react to the dogs' behaviour, and it only started to move after $30 \mathrm{~s}$ passed. So on the second trial dogs may have not been so confident in their choices because they oriented at the UMO during the first trial in vain, as it did not react to their behaviour but started to move independently from it after 30 seconds at a random time. We know from our previous study that dogs initiate interactions with an UMO that behaves interactively with them (if the partner starts to move when the dog looks at it) (Gergely et al. 2013) 
These results support previous findings by Horn et al. (2012), although importantly dogs in the present study discriminated the role of both partners equally well. We assume that this stronger effect was due to the improved methodology used in this study. The use of robots enables researchers to control more attributes of the interaction and to better identify which aspects of the agent's behaviour does the subject recognize. In addition to this, it makes differentiating between the various characteristics and skills.(Ladu et al. 2015)

Dogs rapidly (after 5-5 trials) learned to discriminate between the two UMOs and solicited the appropriate UMO in the specific situation. This rapid learning is quite interesting given earlier reports of how many trials the dogs needed to reach a similar performance in other contexts. For example, in non-interactive contexts involving traditional methods of discrimination learning dogs may need 20 to 300 trials for achieving reliable performance (Milgram et al. 1994). Milgram et al. (1994) reported that laboratory beagles learned to associate the location of food on the basis of an object placed nearby. However, on average these dogs needed about 400 trials to learn the discrimination task. Dogs also displayed difficulties in finding a hidden food indicated solely by the presence of a physical marker (object) in a two-way choice task (e.g. Agnetta et al. 2000; Riedel et al. 2006). For example, dogs did not associate the place of the marker and the location of the hidden food after more than 70 trials (Agnetta et al. 2000). In contrast, such discrimination develops faster if dogs learn in a social context. Dogs learned to rely on a novel beacon for finding hidden food even after 20 trials when the indicating object was manipulated by a human experimenter (Agnetta et al. 2000).

The possibility for interacting with inanimate objects could also facilitate dogs' interpretation of the situation as being social. This can be seen in the emergence of social behaviours if the situation resembles interaction with humans. Jenkins et al. (1978) trained dogs to approach a flashing lamp which indicated the presence of a reward. After repeated interactions the dogs started to show social behaviours toward the lamp (e.g. barking, play bow, tail wagging). Thus, for the dogs the light did not only signal the arrival of food but the dogs also reacted to it as if it were a social partner. This observation was extended by Gergely et al. (2013) who showed that social interaction with an UMO elicit social behaviours in dogs that are also displayed toward humans in similar situations (feeding context).

Dogs in the present experiment could have also recognised the parallels between their everyday interactions with humans (helping to obtain inaccessible food) and the current interaction with the UMOs. This interactive aspect could facilitate the discrimination between two agents and allowed the family dogs to rapidly identify the appropriate UMO. 
Dogs may have been able to recognise the specific abilities of the UMOs (the car is able to bring out the food moving on the floor, and the crane is able raise the bowl). However, the procedures applied both by Horn et al. (2012) and in the present study do not make it possible to come to a definite conclusion. The more parsimonious interpretation is that dogs associated the action of either UMOs with the specific location where the food was hidden.

There is so far no evidence on dogs' ability to attribute specific physical skills to other agents. All findings to date rather suggest their limitation to specific physical regularities, such as "connectedness" or "solidity". For example, Range et al. (2012) reported that dogs did not spontaneously show any preference to a string which was connected to reward over an unconnected one. Dogs did not seem to be able to solve problems in which objects could not pass through a barrier (Müller et al 2014). Although not conclusive, at present this makes also unlikely that dogs have the mental ability to relate different physical skills to other agents.

Despite the limitation of the present study, the utilisation of UMOs has many advantages in studies exploring social problem solving (e.g. Abdai et al. 2015; Gergely et al. 2015). Dogs have no experience with UMOs, thus they are not influenced by previous experience what is the case if human are used as social partners in such experiments. Different sets of UMOs can be used to test dogs' ability to generalise from one interactive agent to another one and how this performance may depend on dogs' previous experience and physical similarity between UMOs. The different abilities of the UMOs were determined by physical constraints, i.e. the car was unable to use the top hole in the absence of a telescopic boom while the size of the crane limited its access to the hidden food through the front hole. The utilisation of such differences could be used in future studies to examine whether dogs are able to recognize the abilities of the robotic agents. Finally, it is generally difficult in the case of a human partner, as the differentiation of ability, willingness and the specific characteristics pose a big challenge but the deployment of UMOs offers possibilities for the differentiation of these concepts.

We conclude that even after a short experience dogs are able to choose the appropriate helping partner when facing a certain problem. The rapid adjustment to the social situation can be explained by dogs' generalisation ability which rested on the similarities experienced with regard to food in human-dog and UMO-dog interactions.

\section{Acknowledgements}


This research was supported by the Swiss National Science Foundation (SNSF) Sinergia project SWARMIX (project number CRSI22 133059) and MTA-ELTE Comparative Ethology Research Group (MTA 01 031) and OTKA K 112138.

The authors are grateful to the owners for participating in the experiment.

\section{References}

Abdai J, Gergely A, Petró E, Topál J,Miklósi Á(2015) An investigation on social representations: Inanimate agent can mislead dogs (Canis familiaris) in a food choice task. PLoS ONE, 10(8): e0134575

Agnetta B, Hare B, Tomasello M (2000) Cues to food location that domestic dogs (Canis familiaris) of different ages do and do not use. Animal Cognition 3:107-112 doi: $10.1007 / \mathrm{s} 100710000070$

Brauer J, Bos M, Call J, Tomasello M (2012) Domestic dogs (Canis familiaris) coordinate their actions in a problem-solving task. Animal Cognition 16:273-285 doi: 10.1007/s10071-012-0571-1

Gaunet F (2008) How do guide dogs of blind owners and pet dogs of sighted owners (Canis familiaris) ask their owners for food? Animal Cognition 11:475-483 doi: 10.1007/s10071-008-0138-3

Gaunet F (2010) How do guide dogs and pet dogs (Canis familiaris) ask their owners for their toy and for playing? Animal Cognition 2:311-323 doi: 10.1007/s 10071-009-0279-z

Gaunet F, Massioui F (2014) Marked referential communicative behaviours, but no differentiation of the "knowledge state" of humans in untrained pet dogs versus 1-yearsold infants. Animal Cognition 17:1137-1147 doi: 10.1007/s10071-014-0746-z

Gergely A, Petró E, Topál J, \& Miklósi Á (2013) What are you or who are you? The emergence of social interaction between dog and an Unidentified Moving Object (UMO). PLoS ONE 8:e72727. doi:10.1371/journal.pone.0072727

Gergely A, Abdai J., Petró E., Kosztolányi A, Topál J, Miklósi Á (2015) Dogs rapidly develop socially competent behaviour while interacting with a contingently responding self-propelled object. Animal Behaviour, 108: 137-144 .

Horn L, Virányi Zs, Miklósi Á, Huber L, Range, F (2012) Domestic dogs (Canis familiaris) flexibly adjust their human-directed behaviour to the actions of their human partners in a problem. Animal Cognition 15:57-71 doi: 10.1007/s10071-011-0432-3

Jenkins H M, Barrera F J, Ireland C, Woodside B (1978) Signal centred action patterns of dogs in appetitive classical conditioning. Learning and Motivation 9:272-296 doi:10.1016/0023-9690(78)90010-3 
Kaminski J, Neumann M, Brauer J, Call J, Tomasello M (2011) Dogs, (Canis familiaris) communicate with humans to request but not to inform. Animal Behaviour 82:651-658 doi: 10.1016/j.anbehav.2011.06.015

Krause J, Winfield A F T, Deneubourg J L (2011) Interactive robots in experimental biology. Trends and Evolution 26:369-375 doi: 10.1016/j.tree.2011.03.015

Ladu F, Bartolini T, Panitz S. G, Chiarotti F, Butail S, Macri S, Porfiri M (2015) Live predators, robots, and computer-animated images elicit differential avoidance responses in zebrafish, Zebrafish 12: 205-214

Lakatos G, Soproni K, Dóka A, Miklósi Á (2009) A comparative approach to dogs' (Canis familiaris) and human infants' comprehension of various forms of pointing gestures. Animal Cognition 12:621-631 doi: 10.1007/s10071-009-0221-4

Melis A P, Hare B, Tomasello M (2006) Chimpanzees recruit the best collaborators. Science 311:1297-1300 doi: 10.1126/science.1123007

Miklósi A, Polgárdi R, Topál J, Csányi V (2000) Intentional behaviour in dog-human communication: an experimental analysis of "showing" behaviour in the dog Animal Cognition 3:159-166 doi: 10.1007/s100710000072

Milgram N. W, Head E, Weiner, E, Thomas E (1994) Cognitive functions and aging in the dog: Acquisition of nonspatial visual tasks. Behavioural Neuroscience108:57 - 68 doi: 10.1037/0735-7044.108.1.57

Range F, Möslinger H, Virányi Zs (2011) Domestication has not affected the understanding of mean-end connection in dogs. Animal Cognition doi: 10.1007/s10071012-0488-8

Riedel J, Buttelmann D, Call J, Tomasello M (2006) Domestic dogs (Canis familiaris) use a physical marker to locate hidden food. Animal Cognition 9:27-35 doi: 10.1007/s10071-005-0256-0

Spinello C, Macri S, Porfiri M (2013) Acute ethanol administration affects zebrafish preference for a biologically-inspired robot", Alcohol 47(5), 391-398

Tapp P D, Siwak C T, Estrada J, Head E, Mugenburg B A, Cotman C W, Milgram N W(2003) Size and reversal learning in the beagle dog as a measure of executive function and inhibitory control in aging. Learning and Memory 10:64-73 doi:

$10.1101 / \mathrm{lm} .54403$

Topál J, Kubinyi E, Gácsi M, Miklósi Á (2005) Obeying social rules a comparative study on dogs and humans. Journal and Cultural and Evolutionary Psychology 223-243 doi: 10.1556/JCEP.3.2005.3-4.1 
Virányi Zs, Topál J, Miklósi Á, Csányi V (2006) A nonverbal test of knowledge attribution: a comparative study on dogs and children. Animal Cognition 9:13-26 doi: $10.1007 / \mathrm{s} 10071-005-0257-\mathrm{z}$ 
465 Tables

466

467 Table 1: The use of doors by the car and the crane during the learning phase, and the doors in 468 front of which the car and the crane was standing in the test phase (FH: the car could access 469 the food through the front hole; TH: the crane could access the food through the top hole) The 470 number of dogs and their ages is provided for each testing condition separately.

\begin{tabular}{|c|c|c|c|c|c|c|c|c|}
\hline & \multicolumn{4}{|c|}{$\underline{\text { Same door condition }}$} & \multicolumn{4}{|c|}{ Changed door condition } \\
\hline Learning phase & \multicolumn{2}{|c|}{$\begin{array}{l}\text { Car: door A } \\
\text { Crane: door B }\end{array}$} & \multicolumn{2}{|c|}{$\begin{array}{l}\text { Car: door B } \\
\text { Crane: door A }\end{array}$} & \multicolumn{2}{|c|}{$\begin{array}{l}\text { Car: door A } \\
\text { Crane: door B }\end{array}$} & \multicolumn{2}{|c|}{$\begin{array}{l}\text { Car: door B } \\
\text { Crane: door A }\end{array}$} \\
\hline $\begin{array}{l}\text { Test Phase } \\
\text { (baited hole) } \\
\end{array}$ & $\frac{1^{\text {st }} \mathrm{FH}}{2^{\text {nd }} \mathrm{TH}}$ & $\frac{1^{\mathrm{st}} \mathrm{TH}}{2^{\text {nd }} \mathrm{FH}}$ & $\frac{1^{\text {st }} \mathrm{FH}}{2^{\text {nd }} \mathrm{TH}}$ & $\frac{1^{\text {st }} \mathrm{TH}}{2^{\text {nd }} \mathrm{FH}}$ & $\frac{1^{\text {st }} \mathrm{FH}}{2^{\text {nd }} \mathrm{TH}}$ & $\frac{1^{\text {st }} \mathrm{TH}}{2^{\text {nd }} F H}$ & $\frac{1^{\mathrm{st}} \mathrm{FH}}{2^{\mathrm{nd}} \mathrm{TH}}$ & $\frac{1^{\mathrm{st}} \mathrm{TH}}{2^{\text {nd }} \mathrm{FH}}$ \\
\hline $\begin{array}{l}\frac{\mathrm{N} \text { of dogs (males }}{\text { \&females) }} \\
\frac{\text { Mean age } \pm \mathrm{SD}}{\text { (years) }}\end{array}$ & $\frac{\frac{N=12}{7 \& 5}}{\frac{4 \pm 2.2}{}}$ & & $\frac{\frac{N=12}{3 \& 9}}{\underline{3.6 \pm 2.3}}$ & & $\frac{\frac{N=12}{3 \& 9}}{\frac{3.2 \pm 2}{\underline{4.2}}}$ & & $\frac{\frac{\mathrm{N}=12}{7 \& 5}}{\underline{7.2 \pm 2.2}}$ & \\
\hline
\end{tabular}


Figure legends

473

474 Figure 1 (a) The wooden box with two holes and the test partners (UMOs) and the bowl.

475 Arrows indicate the hole used by the car or the crane during the training and testing for

476 retrieving the food; (b) Two magnets were attached to the bowl by the means of which the

477 UMOs could it get out from the wooden box.

478

479

\section{Figure 1a}

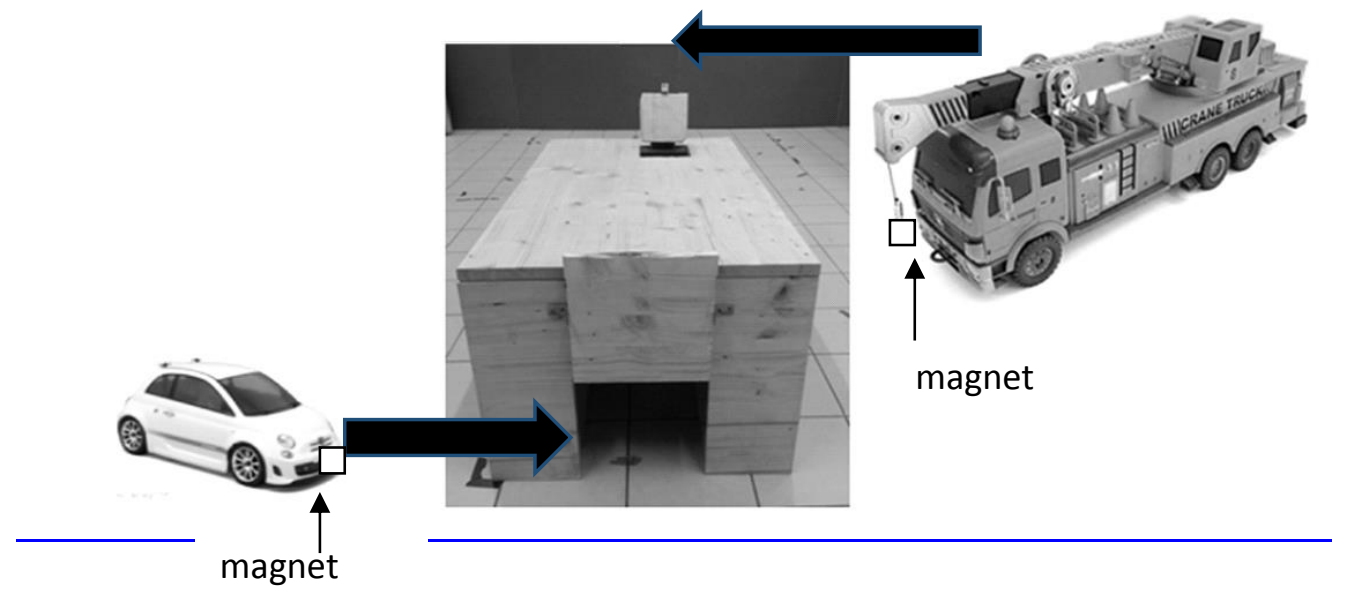

\section{Figure 1b}

482

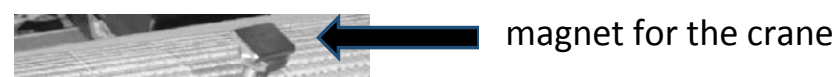

487 Figure 2 Experimental lay out indicating the paths of movement for the UMOs. Dashed lines 488 show the paths of the car from Door A or B to the front hole and then to the dog. Dotted lines 489 show the paths of the crane from Door A or B to the top hole and then to the dog. 
$\mathrm{O}=$ owner's location; $\mathrm{D}=$ dogs' location, $\mathrm{E} 2 / 3=$ experimenters' 2 and 3 location; $\mathrm{B}=$ location

491 of the wooden box; $\mathrm{FH}=$ front hole, $\mathrm{TH}=$ top hole, $\mathrm{P} 1 \& \mathrm{P} 2=$ parking places of the UMOs

492 outside of the experimental room, $\mathrm{T}$ with triangle=positions of the UMOs during test trials;

493 Door A and Door B with guillotine openings.

494

495

Figure 2

496

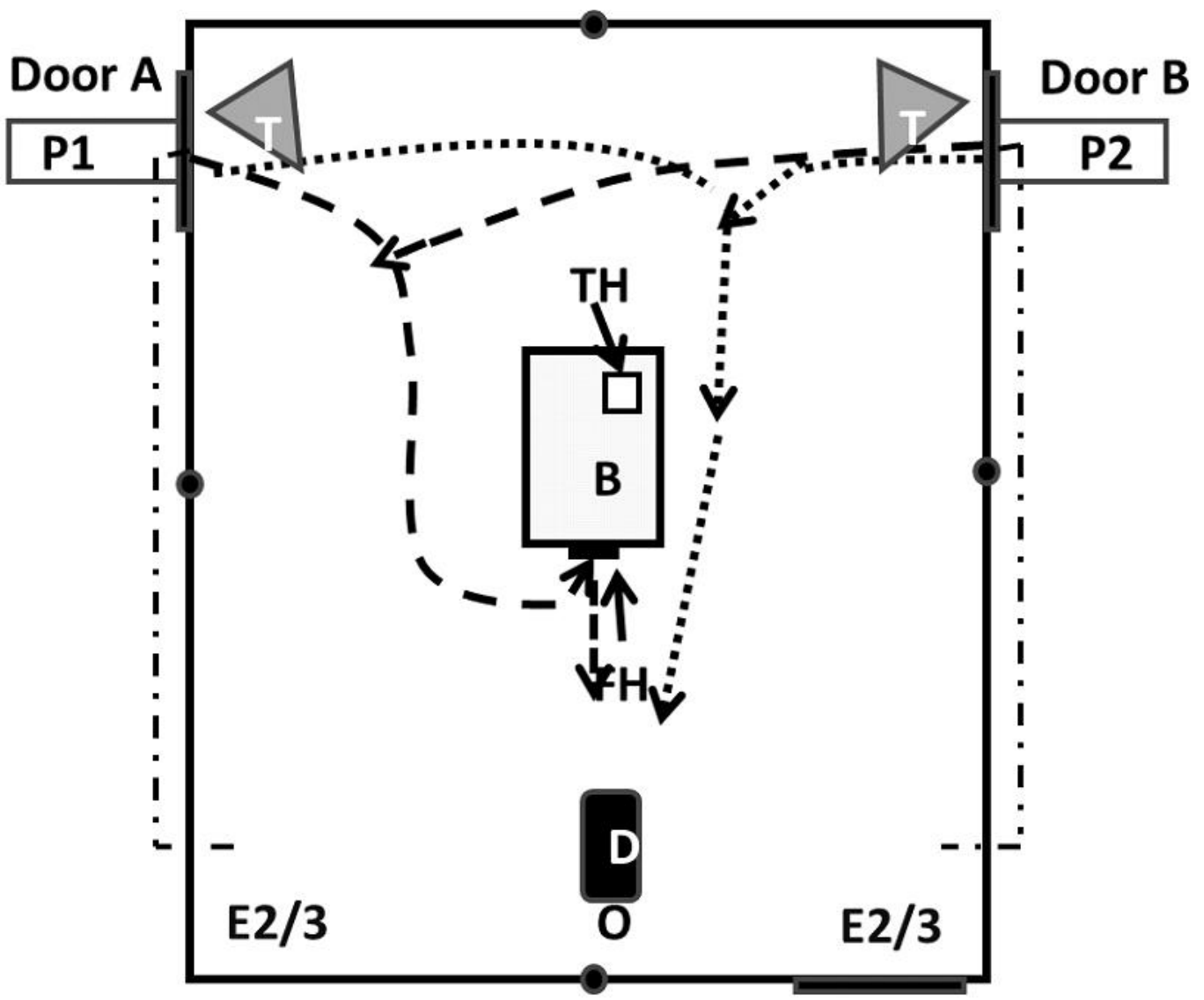

497

Door C

498

499

500

Figure 3 Experimental arrangements from different views. (a) and (b) Starting arrangement of the learning phase; (c) Staring arrangement of the testing phase with both UMOs standing in front of the doors.

503

504

Figure 3 

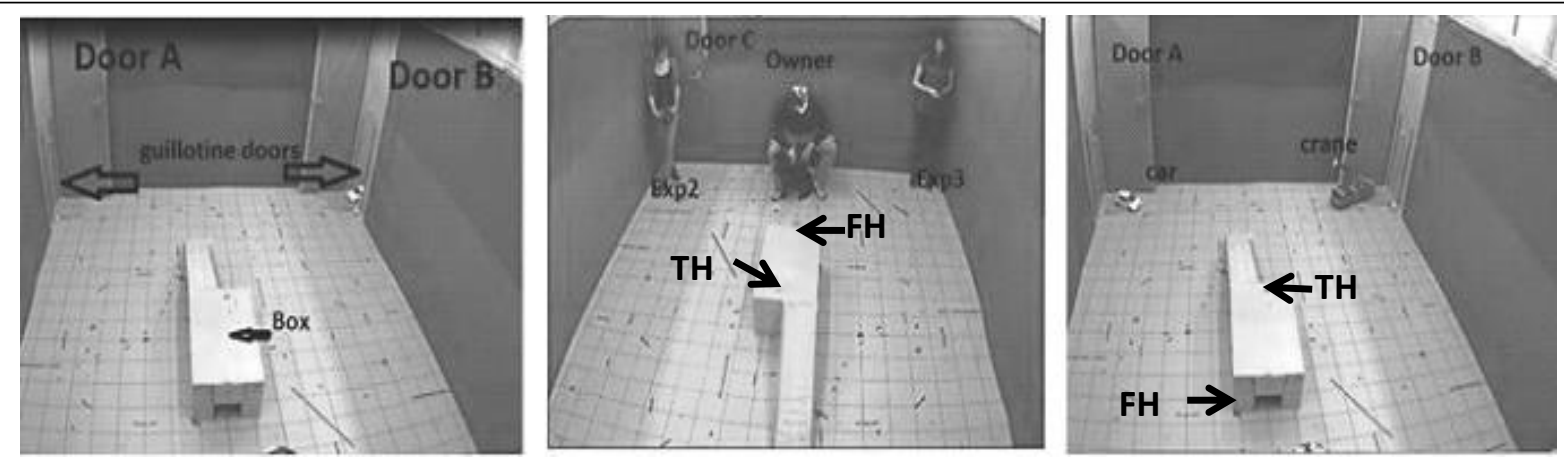

507

508

509

510 Figure 4 Proportion of dogs' correct choices for 'First look at', 'First approach to' and 'First

511 touch' of the UMOs in test trials. Dotted line indicates chance level $(0.5) * \mathrm{p}<0.05$

512 Figure 4

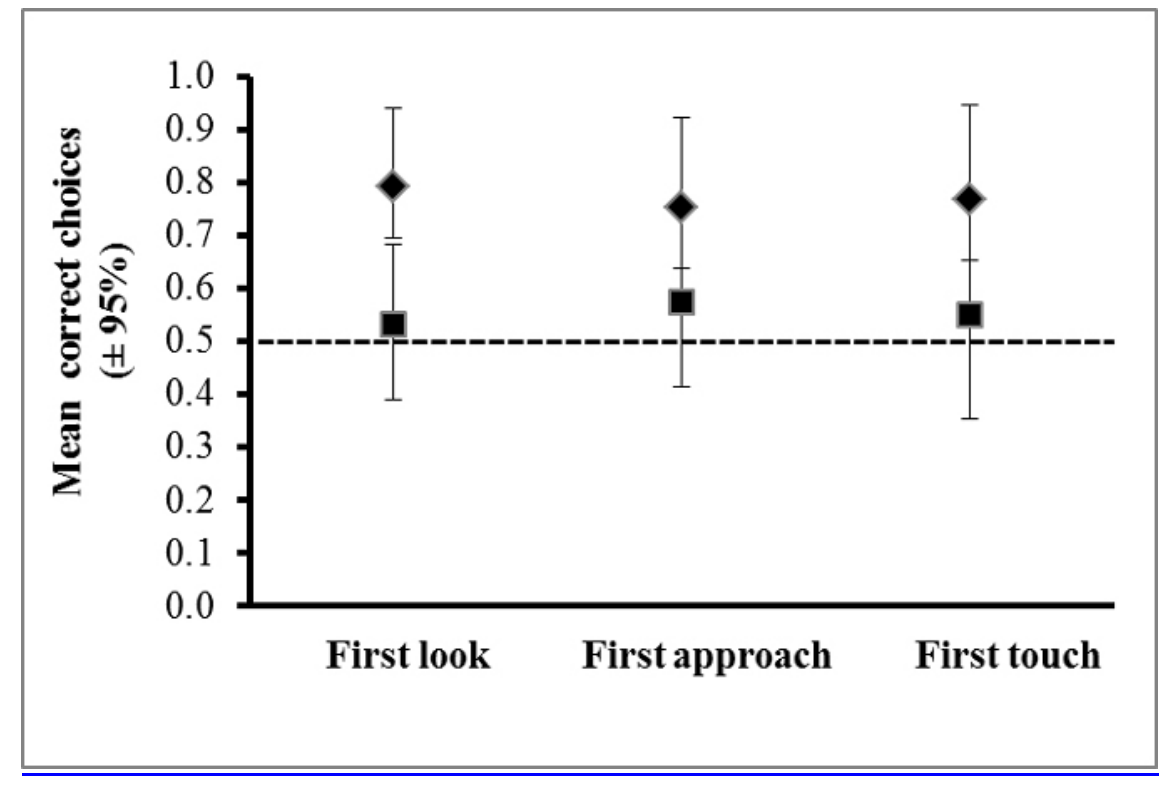

trial 1

513

trial 2

514 
515 
516 
518 
